\title{
Facile preparation of palygorskite/chitin nanofibers hybrids nanomaterial with remarkable adsorption capacity
}

\author{
Stanley Chinedu Mamah ${ }^{\mathrm{a}, \mathrm{b}}$, Pei Sean Goh ${ }^{\mathrm{a}}$, Ahmad Fauzi Ismail ${ }^{\mathrm{a}, *}$, \\ Mohamed Afizal Mohamed Amin ${ }^{\mathrm{a}}$, Nor Akalili Ahmad ${ }^{\mathrm{a}}$, Nur Diyana Suzaimi ${ }^{\mathrm{a}}$, \\ Yusuf Olobode Raji ${ }^{\text {a,c }}$ \\ a Advanced Membrane Technology Research Centre, School of Chemical and Energy Engineering, Universiti Teknologi Malaysia, 81310 Skudai, Johor, Malaysia \\ ${ }^{\mathrm{b}}$ Department of Chemical Engineering, Alex Ekwueme Federal University, Ebonyi State, Nigeria \\ ${ }^{\mathrm{c}}$ Department of Chemical Engineering, Abubakar Tafawa Balewa University, Bauchi, Nigeria
}

\section{A R T I C L E I N F O}

\section{Keywords:}

Chitin nanofiber

Palygorskite

Hybrid

Characterization

Adsorption capacity

\begin{abstract}
A B S T R A C T
A novel hybrid nanomaterials comprising of palygorskite (PAL) and chitin nanofibers (ChNFs) was successfully synthesized via simple, green and cost effective technology/method and for the first time used in adsorption process. Shrimp sourced chitin is a biopolymer endowed with amino and hydroxyl groups suited for adsorption of metals while PAL is hydrophilic, cheap and eco-friendly material. The hybridization of PAL and ChNFs was achieved under the collision and shear force of ball mill. Morphological analysis showed that ChNFs were attached on PAL leading to enhanced stability of the hybrid dispersion. PAL/ChNFs was used as an adsorbent for $\mathrm{Pb}(\mathrm{II})$ ion metal removal. The hybrid adsorbent showed a remarkable adsorption capacity $\left(\mathrm{q}_{\mathrm{e}}\right)$ of $53.7 \mathrm{mg} \mathrm{\textrm {g } ^ { - 1 }}$ and removal efficiency of $92.9 \%$. This water dispersible PAL/ChNFs holds great potential in different water treatment related applications that require materials with high hydrophilicity, anti-fouling, biocompatibility, adsorption as well as mechanical properties.
\end{abstract}

\section{Introduction}

Water is a major source in the global world as every living thing needs water for survival. The unprecedented population growth and industrialization have released various types of contaminants such as dyes, heavy metals, pharmaceutical organic and inorganic pollutants that have a striking negative impact on the ecosystem [1-3]. It has given rise to eutrophication which leads to algal blooms with detrimental impacts on aquatic and human life.

The removal of pollutants like lead is progressively global concern that demands efficient remediation approach due to the enormous quantities of contaminants steadily being generated [4]. Lead is commonly present in the electronic waste. There is prevalence usage of lead in electrical industries, fungicides and antifouling agents. Consumption of high lead concentration polluted water results in intellectual disability in children leading to developmental delay as well as chronic damage to the nervous system [5]. Hence, removal of lead-containing wastewater is crucial before discharging into the environment [6].

Execution of regulations to minimize the concentration of lead in wastewater has led to the establishment of different lead treatment technologies like adsorption [7], reverse osmosis [8], ion exchange [9] and electrocoagulation [10]. These chemical treatments have certain limitations for practical applications [11]. For instance, methods such as electrocoagulation and reverse osmosis are capital intensive, with sludge generation as well as residual toxicity. Thus, development of low cost and eco- friendly adsorbent will be a viable option. The use of nanoparticles based adsorbents is a promising and attractive alternative for wastewater treatment due to inherent advantages like eco-friendly characteristic, cost effectiveness, ease of operation and speedy adsorption rate [12]. Development of an efficient adsorbent gives room for effectiveness of adsorption technique [13].

Many adsorbents have been developed for lead via various synthesis and modification methods. Nevertheless, insufficient removal associated with the existing adsorbents have limited their practical applications. Therefore, the design of effective adsorbent for lead-containing waste treatment is still highly required in this area.

Clays are known to be one of the ideal constituents for thin nanocomposite owing to their high interfacial reactivity and inherent hydrophilicity [14]. In general, clays are mainly silicates or alumina silicates, which comprise of aluminium or magnesium, silicon, oxygen

\footnotetext{
* Corresponding author.

E-mail address: afauzi@utm.my (A.F. Ismail).
} 